\title{
Review
}

Korean J Obes 2016 September;25(3):109-114

http://dx.doi.org/10.7570/kjo.2016.25.3.109

pISSN 2383-899X elSSN 2234-7631

\section{Food Intake and Thermogenesis in Adipose Tissue}

\author{
Tsuyoshi Goto ${ }^{1,2}$, Minji Kim ${ }^{1}$, Haruya Takahashi ${ }^{1}$, Nobuyuki Takahashi ${ }^{1,2, \#}$, Teruo Kawada ${ }^{1,2, *}$ \\ ${ }^{1}$ Laboratory of Molecular Function of Food, Division of Food Science and Biotechnology, Graduate School of Agriculture, Kyoto University, Uji, Japan; ${ }^{2}$ Research Unit \\ for Physiological Chemistry, The Center for the Promotion of Interdisciplinary Education and Research, Kyoto University, Kyoto, Japan
}

Brown adipose tissue (BAT) is regarded as a key target for developing interventions to prevent and treat obesity and age-related diseases. In addition, uncoupling pro tein 1 (UCP1) - expressing "brown-like" adipocytes, known as beige cells, that develop in white adipose tissue (WAT) under certain physiological conditions and pharmacological stimuli. The effects of ageing on BAT atrophy and functional decline further strengthen the notion that healthy eating habits and food choices play an extremely vital role against reduction in the number of BAT and beige cells as people approach middle age. This review aims to cover recent progress in food items and pharmaceuticals that activate or induce BAT and UCP1.

Key words: Food components, Obesity, Brown adipose tissue, Uncoupling protein, Beige cells, Transient receptor potential (TRP) channels

\section{Introduction}

Diet-induced thermogenesis (DIT) is defined as an increase in body temperature, which is more than the inducible temperature, due to the metabolism of food consumed during a meal. ${ }^{1}$ It has two components: increase in the body heat production due to energy metabolism, by activating the sensory nervous system - the taste and smell of food - and the increase in food digestion and absorption. The former is affected by various factors during food consumption. Recently, "non-energetic food components" such as taste and smell were found to be linked to increase in DIT via sensory nerve stimulation, in addition to the increase due to fat metabolism within brown adipose tissue (BAT). In this review, we will discuss the effect of various factors, along with the food consumption on thermogenesis in adipose tissue.

\section{Adipose tissue and thermogenesis}

Obesity is defined as excessive storage of fat by the adipose tissue in the body. It is composed of white and brown adipocytes (Fig. 1).
White adipocytes form white adipose tissue (WAT), which stores energy, whereas BAT is a "heat-producing tissue", which oxidizes deposited fat and releases the stored energy as heat. Brown adipocytes have many mitochondria, which contain uncoupling protein-1 (UCP1), involved in accelerating heat production by uncoupling ATP synthesis. Heat production is mainly regulated by the sympathetic nervous system. BAT helps to maintain optimum body temperature in a cold environment by generating and dissipating heat from stored energy.

In recent years, it is shown that in healthy ageing humans, reduction of BAT activity might be associated with body fat accumulation in WAT. ${ }^{2}$ These results suggest that, BAT might have a protective role against increase in body fat storage with age. Therefore, BAT is regarded as a key target for developing interventions to prevent and treat obesity and age-related diseases. It is reported that two distinct types of brown adipocytes exist: classical brown adipocytes that arise from muscle-like type progenitors ${ }^{3}$ and UCP1-expressing "brown-like" adipocytes, known as beige cells, that develop in WAT under certain physiological conditions (such as cold exposure) and pharmacological stimuli (such as beta-adrenergic agonists). ${ }^{4,5}$ Beige
Corresponding author Teruo Kawada (iD http://orcid.org/0000-0002-7067-4380 Laboratory of Molecular Function of Food, Division of Food Science and Biotechnology, Graduate School of Agriculture, Kyoto University, 611-0011, Gokasho, Uji, Kyoto, Japan Tel: +81-774-38-3751 Fax:+81-774-38-3752 E-mail: fat@kais.kyoto-u.ac.jp \#Current address: Laboratory of Physiology and Metabolism, Department of Nutritional Science and Food Safety, Faculty of Applied Bioscience, Tokyo University of Agriculture, Setagaya, Tokyo 156-8502 Japan.
Copyright (C) 2016 Korean Society for the Study of Obesity

(a) This is an Open Access article distributed under the terms of the Creative Commons Attribution Non-Commercial License (http://creativecommons.org/licenses/by-nc/4.0/) which permits unrestricted non-commercial use, distribution, and reproduction in any medium, provided the original work is properly cited. 


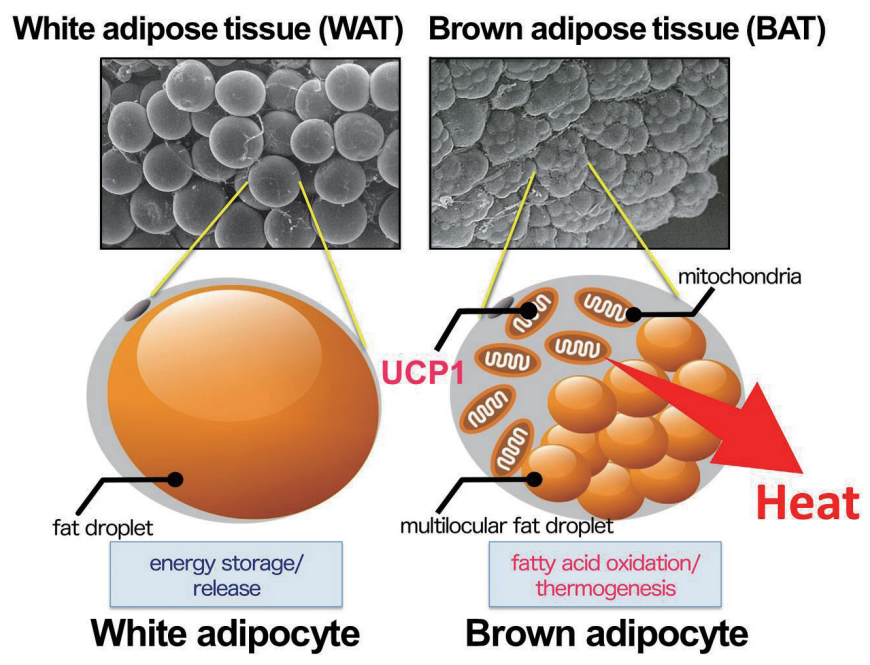

Fig. 1. White adipocyte and brown adipocyte.

cells also reduce in number as people approach middle age; without these metabolizing cells, fat continues to accumulate for decades called a middle-age spread. ${ }^{2,6}$ Obese WAT exhibited a decrease in energy expenditure. ${ }^{7,8}$ However, there is insufficient understanding of the relationship between the reduction in energy expenditure and the induction of beige cell in WAT from the point of ageing.

\section{BAT atrophy and functional decline}

It was elucidated that decreased BAT activity may be associated with accumulation of body fat in WAT with ageing in healthy humans. ${ }^{2}$ It is well known that chronic inflammation leads to metabolic dysfunctions in obese WAT., ${ }^{90} \mathrm{M} 1$ macrophages $(\mathrm{M} \varphi)$ infiltrate WAT via cytokines, mainly monocyte chemoattractant protein-1 (MCP-1), which are released from hypertrophied adipocytes and M1 $\mathrm{M} \varphi$ in obese WAT. These infiltrated $\mathrm{M} \varphi$ are then activated by free fatty acids from hypertrophied adipocytes, resulting in the secretion of proinflammatory cytokines such as tumor necrosis factor-alpha (TNFa). ${ }^{11,12}$

We evaluated that these proinflammatory cytokines - derived from the activated $\mathrm{M} \varphi$ - are one of the inhibitory factors involved in the induction of brown-like adipocytes and UCP1 expression that may lead to a decrease in energy expenditure in obese WAT. We further identified TNF $\alpha$ and interleukin-1 beta (IL-1 $\beta$ ) as possible proinflammatory cytokines involved in the suppression of UCP1 expression in adipocytes. ${ }^{13-15}$ These reports suggest a plausible mechanism of UCP1 transcriptional repression in white adipocytes, in obesity and its related disorder, diabetes. Investigating such a mechanism will provide insights into the physiological and pathological regulation of UCP1 and help develop a solution to overcome the decrease in energy expenditure under obese conditions.

\section{Sensory stimulation by food intake and thermogenesis}

Eating not only involves the ingestion of food, but also stimulates the gustatory, olfactory, and somatic senses. Besides the joy, eating also influences other sensory activities. Recently, the signals released in response to sensory stimulation, as well as the physiological phenomena associated with food intake, digestion, absorption, and various hormone secretions were studied. ${ }^{16}$ The effect of food components on the nervous and hormonal regulatory system has been largely unrecognized, until now.

However, the significant role of "non-nutrient" food factors in thermogenesis has been established. ${ }^{17}$ Le Blanc et al. ${ }^{18}$ showed that "palatability" of food acts as a signal affecting the central nervous system, causing catecholaminergic thermogenesis in humans and dogs.

\section{Lipid intake and thermogenesis}

Even though the phenomenon of accelerated thermogenesis caused by lipid intake has been observed for a long time, factors affecting this mechanism are poorly understood. In rats, lipid digestion leads to an increase in sympathetic nerve activity causing thermogenesis: it induces a cessation of treatment with cholestyramine, a bile acid sequestrant that blocks the digestion and absorption of lipids and is used to treat hypercholesterolemia. Increase in thermogenesis due to lipid intake is signaled either by the release of fatty acids during lipid digestion or secretion of gastrointestinal hormones in response to these fatty acids. ${ }^{19}$

Dietary lipids vary according to their constituent fatty acids, for instance, shortening (containing a high level of saturated fatty acid) and lard (containing a same level of saturated fatty acid and monounsaturated fatty acid) affect the concentration of catecholamine in urine due to an effect on sympathetic nerve. ${ }^{10,20,21}$ Interestingly, UCP1 expression also differs with BAT development. ${ }^{22,23}$ Fatty acid receptor, such as $\mathrm{G}$ protein-coupled receptors (GPCRs) exist in the 
mouth; intraoral recognition of free fatty acids contribute to the depolarization of receptor cells, thereby activating the gastrointestinal organs involved in the digestion and sensing of lipids through the sympathetic nervous system. ${ }^{24}$ Because the intraoral mechanism of lipid recognition is linked to lipid palatability $\operatorname{control}^{25,26}$, lipid sensory stimulation and the subsequent central phase stimulation are closely associated with the lipid-related thermogenesis.

Recently, we reported that the intake of fish oil containing eicosapentaenoic acid (EPA) and docosahexaenoic acid (DHA) enhances energy utilization by inducing UCP1 in both BAT and WAT containing beige cells through transient receptor potential vanilloid 1 (TRPV1) channel-sympathetic nerve system (SNS) and may prevent obesity and its related metabolic disorders. ${ }^{27}$ Interestingly, we found that a linoleic acid metabolite, produced by gut lactic acid bacteria, enhances energy metabolism by activation of TRPV1 (under submission).

\section{Plant derived compounds and thermogenesis}

In 1980s, we studied the effect of spices as a factor in enriching the palatability of food. Pungent ingredients in spices such as pepper induce the acceleration of thermogenesis: "pungent ingredient-in-

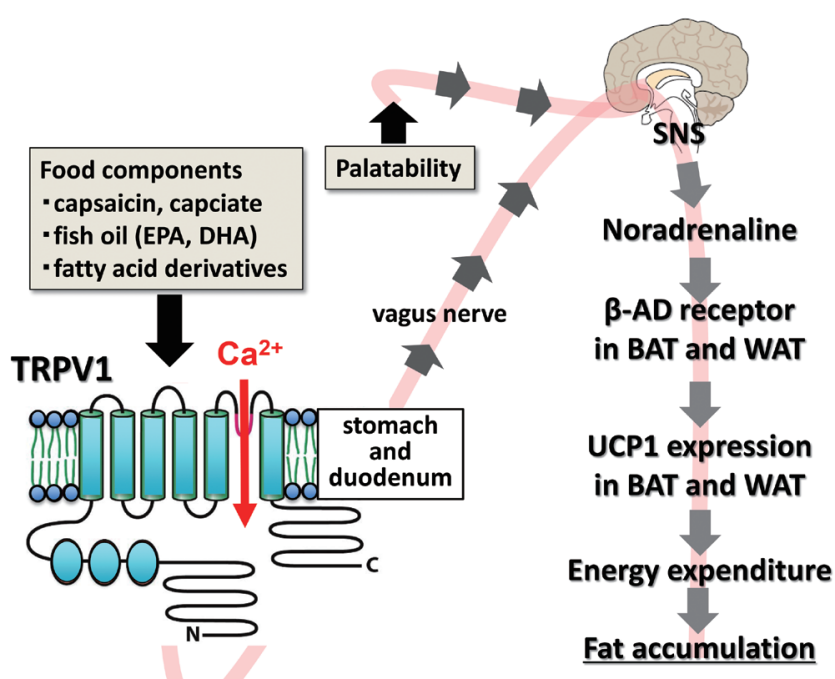

Fig. 2. Schematic summary of anti-obesity effect of food intake via TRPV1 activation.

EPA, eicosapentaenoic acid; DHA, docosahexaenoic acid; TRPV1, transient receptor potential vanilloid1; SNS, sympathetic nervous system; $\beta$-AD, $\beta$-adrenergic; UCP1, uncoupling protein 1; BAT, brown adipose tissue; WAT, white adipose tissue. duced thermogenesis". ${ }^{28}$ These pungent ingredients activate the sympathetic nervous system in the intraoral and digestive tract, resulting in the secretion of adrenaline from the adrenal glands. The adrenergic signal causes the augmentation of energy metabolism and reduction of body fat ${ }^{29-31}$, by activating and strengthening of BAT through sympathetic nerve activity.

It is reported that the most effective physiological condition for activating BAT in humans is exposure to cold. ${ }^{32,33}$ This process is postulated to occur through the following mechanism: cold stimulus received by sensory nerves activates the sympathetic nervous system via hypothalamus, which then activates BAT through beta-adrenergic receptors on brown adipocytes. This is considered a type of "central phase stimulus". Moreover, based on the concept of this process Saito et al. focused on the food component agonist sources of TRPV1, a type of transient receptor potential (TRP) channel. Interestingly, capsinoids, a nonpungent constituent of pepper, and parad$\mathrm{ol}$, a constituent of ginger, activate human BAT and increase the expenditure of energy. ${ }^{34,35}$ These results suggest that the continuous stimulation of TRP channel by food components can be a simple and effective means of expression, functional maintenance, and augmentation of BAT via the sympathetic nervous system (Fig. 2). The dietary compounds responsible for BAT and UCP1 activation are

Table 1. Dietary compounds of activators and inducers on BAT and UCP1

\begin{tabular}{ll}
\hline Foods (functional compounds) & \multicolumn{1}{c}{ Estimated action mechanism } \\
\hline Fatty acids & UCP-1 proton conductance \\
Vitamin A (all-trans retinoic acid) & UCP-1 proton conductance \\
Sweetener (sucrose) & sympathetic nervous system \\
Sweetener (saccharin) & sympathetic nervous system \\
Mustard (pungency: ally isothiocyanate) & sympathetic nervous system \\
Hot pepper (pungency: capsaicin) & TRPV1-sympathetic nervous system \\
Hot pepper (non-pungency: capsiate) & TRPV1-sympathetic nervous system \\
Ginger (pungency: parado) & sympathetic nervous system \\
Coffee (caffeine) & sympathetic nervous system \\
Olive (oleuropein) & sympathetic nervous system \\
Grapefruit (limonene) & sympathetic nervous system \\
Allonear berry (allonear anthocyanin) & sympathetic nervous system \\
Brown Seaweeds (carotenoid: fucoxanthin) & unknown \\
Fish oil (EPA, DHA) & PPARs ligands \\
& sympathetic nervous system \\
& UCP1-1 proton conductance? \\
1-Monoacylglycerol & TRPV1-sympathetic nervous system \\
Fatty acid derivative (CLA) & PPARs ligands? \\
& UCP-1 proton conductance?
\end{tabular}

TRPV, transient receptor potential vanilloid; EPA, eicosapentaenoic acid; DHA, docosahexaenoic acid; PPAR, peroxisome proliferator-activated receptor; CLA, conjugated linoleic acid. 
summarized in Table 1.

A few other food compounds cause an increase in BAT activity by stimulating adrenaline and noradrenaline secretions, through the interplay between the sympathetic nervous system and adrenal glands. For example, when rats were fed oleuropein, a compound from olive oil, UCP1 expression increased significantly after 28 days; an increase in BAT function, decrease in body weight, and fat pad was also observed. ${ }^{36}$ Oleuropein, a polyphenolic compound, is abundantly found in extra-virgin olive oil and imparts bitterness. Polyphenols from Kaempferia parviflora, a member of the ginger family, enhances UCP1 expression in BAT and decreases fat pad in mouse ${ }^{37}$ and increases whole-body energy expenditure in humans. ${ }^{38}$

There are many plant-derived compounds (phytochemicals) such as polyphenols (more than 8000 kinds) and carotenoids (more than 600 kinds) in food. Many epidemiological studies suggest that consumption of polyphenol-rich or carotenoid-rich foods have a beneficial effect on diabetes, obesity, and cardiovascular risk factors. Phytochemicals exert their effect through the antioxidant and anti-inflammatory mechanisms. ${ }^{39-42}$ This suggests that phytochemicals in food can act as anti-inflammatory factors, thereby decreasing BAT atrophy and their subsequent functional decline. Further longitudinal studies on human subjects are necessary to confirm the above idea.

\section{Conclusion}

Many fatty acids and plant-derived compounds from food activate and strengthen classical brown adipocytes in BAT and beige cells in BAT and WAT through the TRP-induced activation of the sympathetic nervous system via sensory stimulation. The effects of ageing on BAT atrophy and functional decline further strengthen the notion that healthy eating habits and food choices play an extremely vital role against reduction in the number of BAT and beige cells as people approach middle age. In the fight against obesity and metabolic diseases in an ageing society, the generation of food items and development of pharmaceuticals that activate or induce BAT and $\mathrm{UCP} 1$ is warranted.

\section{Conflicts of Interest}

The authors declare no conflict of interest.

\section{Acknowledgments}

The authors thank Sayoko Shinto for secretarial assistance. This work was supported by Grants-in-Aid for Scientific Research from the Ministry of Education, Culture, Sport, Science and Technology of Japan (16H02551, 15K07441, 16K07734, and 22228001) and the Research Project on Development of Agricultural Products and Foods with Health-promoting benefits (NARO) (2013-A-10), Japan.

\section{References}

1. Rothwell NJ, Stock MJ. A role for brown adipose tissue in dietinduced thermogenesis. Nature 1979;281:31-5.

2. Yoneshiro T, Aita S, Matsushita M, Okamatsu-Ogura Y, Kameya T, Kawai Y, et al. Age-related decrease in cold-activated brown adipose tissue and accumulation of body fat in healthy humans. Obesity (Silver Spring) 2011;19:1755-60.

3. Seale P, Bjork B, Yang W, Kajimura S, Chin S, Kuang S, et al. PRDM16 controls a brown fat/skeletal muscle switch. Nature 2008;454:961-7.

4. Cousin B, Cinti S, Morroni M, Raimbault S, Ricquier D, Pénicaud L, et al. Occurrence of brown adipocytes in rat white adipose tissue: molecular and morphological characterization. J Cell Sci 1992;103:931-42.

5. Nagase I, Yoshida T, Kumamoto K, Umekawa T, Sakane N, Nika$\mathrm{mi} \mathrm{H}$, et al. Expression of uncoupling protein in skeletal muscle and white fat of obese mice treated with thermogenic beta 3 -adrenergic agonist. J Clin Invest 1996;97:2898-904.

6. Graja A, Schulz TJ. Mechanisms of aging-related impairment of brown adipocyte development and function. Gerontology 2015;61: 211-7.

7. Choo HJ, Kim JH, Kwon OB, Lee CS, Mun JY, Han SS, et al. Mitochondria are impaired in the adipocytes of type 2 diabetic mice. Diabetologia 2006;49:784-91.

8. Wlodek D, Gonzales M. Decreased energy levels can cause and sustain obesity. J Theor Biol 2003;225:33-44.

9. Guilherme A, Virbasius JV, Puri V, Czech MP. Adipocyte dysfunctions linking obesity to insulin resistance and type 2 diabetes. Nat Rev Mol Cell Biol 2008;9:367-77.

10. Hotamisligil GS. Inflammation and metabolic disorders. Nature 2006;444:860-7. 
11. Suganami T, Nishida J, Ogawa Y. A paracrine loop between adipocytes and macrophages aggravates inflammatory changes: role of free fatty acids and tumor necrosis factor alpha. Arterioscler Thromb Vasc Biol 2005;25:2062-8.

12. Xu H, Barnes GT, Yang Q, Tan G, Yang D, Chou CJ, et al. Chronic inflammation in fat plays a crucial role in the development of obesity-related insulin resistance. J Clin Invest 2003;112:1821-30.

13. Sakamoto T, Takahashi N, Sawaragi Y, Naknukool S, Yu R, Goto T, et al. Inflammation induced by RAW macrophages suppresses UCP1 mRNA induction via ERK activation in 10T1/2 adipocytes. Am J Physiol Cell Physiol 2013;304:C729-38.

14. Sakamoto T, Nitta T, Maruno K, Yeh YS, Kuwata H, Tomita K, et al. Macrophage infiltration into obese adipose tissues suppresses the induction of UCP1 expression in mice. Am J Physiol Endocrinol Metab 2016 [Epub ahead of print].

15. Goto T, Naknukool S, Yoshitake R, Hanafusa Y, Tokiwa S, Li Y, et al. Proinflammatory cytokine interleukin- $1 \beta$ suppresses cold-induced thermogenesis in adipocytes. Cytokine 2016;77:107-14.

16. Mattes RD. Physiologic responses to sensory stimulation by food: nutritional implications. J Am Diet Assoc 1997;97:406-13.

17. Power ML, Schulkin J. Anticipatory physiological regulation in feeding biology: cephalic phase responses. Appetite 2008;50:194206.

18. LeBlanc J, Brondel L. Role of palatability on meal-induced thermogenesis in human subjects. Am J Physiol 1985;248:E333-6.

19. Kaufman LN, Young JB, Landsberg L. Effect of protein on sympathetic nervous system activity in the rat. Evidence for nutrientspecific responses. J Clin Invest 1986;77:551-8.

20. Oi-Kano Y, Kawada T, Watanabe T, Koyama F, Watanabe K, Senbongi $\mathrm{R}$, et al. Extra virgin olive oil increases uncoupling protein 1 content in brown adipose tissue and enhances noradrenaline and adrenaline secretions in rats. J Nutr Biochem 2007;18:685-92.

21. Oi Y, Kawada T, Shishido C, Wada K, Kominato Y, Nishimura S, et al. Allyl-containing sulfides in garlic increase uncoupling protein content in brown adipose tissue, and noradrenaline and adrenaline secretion in rats. J Nutr 1999;129:336-42.

22. Kawada T, Kayahashi S, Hida Y, Koga K, Nadachi Y, Fushiki T. Fish (bonito) oil supplementation enhances the expression of uncoupling protein in brown adipose tissue of rat. J Agric Food Chem 1998;46:1225-7.

23. Oudart H, Groscolas R, Calgari C, Nibbelink M, Leray C, Le
Maho Y, et al. Brown fat thermogenesis in rats fed high-fat diets enriched with n-3 polyunsaturated fatty acids. Int J Obes Relat Metab Disord 1997;21:955-62.

24. Mattes RD. Is there a fatty acid taste? Annu Rev Nutr 2009;29:30527.

25. Mizushige T, Inoue K, Fushiki T. Why is fat so tasty? Chemical reception of fatty acid on the tongue. J Nutr Sci Vitaminol (Tokyo) 2007;53:1-4.

26. Pepino MY, Love-Gregory L, Klein S, Abumrad NA. The fatty acid translocase gene CD36 and lingual lipase influence oral sensitivity to fat in obese subjects. J Lipid Res 2012;53:561-6.

27. Kim M, Goto T, Yu R, Uchida K, Tominaga M, Kano Y, et al. Fish oil intake induces UCP1 upregulation in brown and white adipose tissue via the sympathetic nervous system. Sci Rep 2015;5:18013.

28. Kawada T, Sakabe S, Watanabe T, Yamamoto M, Iwai K. Some pungent principles of spices cause the adrenal medulla to secrete catecholamine in anesthetized rats. Proc Soc Exp Biol Med 1988;188:229-33.

29. Kawada T, Watanabe T, Takaishi T, Tanaka T, Iwai K. Capsaicininduced beta-adrenergic action on energy metabolism in rats: influence of capsaicin on oxygen consumption, the respiratory quotient, and substrate utilization. Proc Soc Exp Biol Med 1986;183: 250-6.

30. Kawada T, Hagihara K, Iwai K. Effects of capsaicin on lipid metabolism in rats fed a high fat diet. J Nutr 1986;116:1272-8.

31. Watanabe T, Kawada T, Kurosawa M, Sato A, Iwai K. Adrenal sympathetic efferent nerve and catecholamine secretion excitation caused by capsaicin in rats. Am J Physiol 1988;255:E23-7.

32. Saito M, Okamatsu-Ogura Y, Matsushita M, Watanabe K, Yoneshiro T, Nio-Kobayashi J, et al. High incidence of metabolically active brown adipose tissue in healthy adult humans: effects of cold exposure and adiposity. Diabetes 2009;58:1526-31.

33. Yoneshiro T, Aita S, Matsushita M, Kameya T, Nakada K, Kawai Y, et al. Brown adipose tissue, whole-body energy expenditure, and thermogenesis in healthy adult men. Obesity (Silver Spring) 2011; 19:13-6.

34. Yoneshiro T, Aita S, Kawai Y, Iwanaga T, Saito M. Nonpungent capsaicin analogs (capsinoids) increase energy expenditure through the activation of brown adipose tissue in humans. Am J Clin Nutr 2012;95:845-50.

35. Sugita J, Yoneshiro T, Hatano T, Aita S, Ikemoto T, Uchiwa H, et 
al. Grains of paradise (Aframomum melegueta) extract activates brown adipose tissue and increases whole-body energy expenditure in men. Br J Nutr 2013;110:733-8.

36. Oi-Kano Y, Kawada T, Watanabe T, Koyama F, Watanabe K, Senbongi $\mathrm{R}$, et al. Oleuropein, a phenolic compound in extra virgin olive oil, increases uncoupling protein 1 content in brown adipose tissue and enhances noradrenaline and adrenaline secretions in rats. J Nutr Sci Vitaminol (Tokyo) 2008;54:363-70.

37. Yoshino S, Kim M, Awa R, Kuwahara H, Kano Y, Kawada T. Kaempferia parviflora extract increases energy consumption through activation of BAT in mice. Food Sci Nutr 2014;2:634-7.

38. Matsushita M, Yoneshiro T, Aita S, Kamiya T, Kusaba N, Yamaguchi K, et al. Kaempferia parviflora extract increases whole-body energy expenditure in humans: roles of brown adipose tissue. J
Nutr Sci Vitaminol (Tokyo) 2015;61:79-83.

39. Li T. Vegetables and fruits used to protect health. In: Vegetables and fruits: nutritional and therapeutic values. New York: CRC Press; 2008. p. 202.

40. Hirai S, Takahashi N, Goto T, Lin S, Uemura T, Yu R, et al. Functional food targeting the regulation of obesity-induced inflammatory responses and pathologies. Mediators Inflamm 2010;2010: 367838 .

41. Lee CG, Koo JH, Kim SG. Phytochemical regulation of Fyn and AMPK signaling circuitry. Arch Pharm Res 2015;38:2093-105.

42. Li Y, Goto T, Ikutani R, Lin S, Takahashi N, Takahashi H, et al. Xanthoangelol and 4-hydroxyderrcin suppress obesity-induced inflammatory responses. Obesity (Silver Spring) 2016 [Epub ahead of print]. 